\title{
GELFAND PAIRS AND SPHERICAL FUNCTIONS
}

\section{JEAN DIEUDONNÉ}

Villa Orangini

119 Avenue de Brancolar

06100 Nice, France

(Received April 5, 1979)

This is a summary of the lectures delivered on Special Functions and Linear Representation of Lie Groups at the NSF-CBMS Research Conference at East Carolina University in March 5-9, 1979. The entire lectures will be published by the American Mathematical Society as a conference monograph in Mathematics. KEY WORDS AND PHRASES. Lie Groups, Linear Representations, Spherical Functions, Special Functions, and Fourier Trans forms.

1980 MATHEMATICS SUBJECT CLASSIFICATION CODES. 33A45, 33A65, 43A65, $22 E 10$.

Since the works of E.Cartan and H.Weyl around 1930, it has been recognized that many of the "special functions" introduced in Analysis since the eighteenth century are closely related to the theory of linear representations of Lie groups which "explains" many of their properties. Among the most interesting are the spherical functions; their theory generalizes both the classical Laplace "spherical harmonics" and commutative harmonic analysis, and they play an important part in the modern theory of infinite dimensional linear representations of Lie groups 
(the so-called "noncommutative harmonic analysis").

Recall that a locally compact group $G$ is called unimodular if its left Haar measure is also invariant under right translations; it is then also invariant under the symmetry $x \mapsto x^{-1}$. Examples of noncommutative unimodular groups are compact groups and semi-simple Lie groups.

For a unimodular group $G$ with Haar measure $m_{G}$, the convolution $f * g$ of two functions $f, g$ in $L^{1}\left(G, m_{G}\right)$ is defined by

$$
(f * g)(x)=\int_{G} f\left(x t^{-1}\right) g(t) d m_{G}(t)
$$

and belongs to $\mathrm{L}^{1}(\mathrm{G})$; for that operation, $\mathrm{L}^{1}(\mathrm{G})$ becomes a Banach algebra for the usual norm, but if $G$ is not commutative, $L^{1}(G)$ is not commutative. There is, however, a remarkable situation involving a compact subgroup $K$ of $G$ and leading to a commutative Banach algebra. One considers in $L^{1}(G)$ functions which are invariant under both left and right translations by elements of $\mathrm{K}$, in other words

$$
f(t x)=f(x t)=f(x) \quad \text { for all } t \in \mathbb{K}
$$

(equality being understood for almost all $x \in G$ ); the subspace of $L^{1}(G)$ consisting of these functions is written $\mathrm{L}^{1}(\mathrm{~K} \backslash \mathrm{G} / \mathrm{K})$; it is a closed subalgebra of the Banach algebra $L^{1}(G)$. In general, that subalgebra is not commutative; we say that $(G, K)$ is a Gelfand pair if $L^{1}(K \backslash G / K)$ is commutative. This is obviously the case when $G$ itself is commutative; but the importance of the notion stems from Gelfand's theorem: Let $\sigma: G \rightarrow G$ be an involutive automorphism of the locally compact unimodular group $\mathrm{G}$, and let $\mathrm{K}$ be the closed subgroup of elements of $\mathrm{G}$ invariant by $\sigma$. Suppose that : $1^{\circ} \mathrm{K}$ is compact ; $2^{\circ}$ each $\mathrm{x} \in \mathrm{G}$ can be written in at least one way as $\mathrm{x}=\mathrm{yz}$, with $\sigma(\mathrm{y})=\mathrm{y}$ and $\sigma(\mathrm{z})=\mathrm{z}^{-1}$. Then $(G, K)$ is a Gelfand pair.

A trivial case of Gelfand pair consists in a commutative locally compact group $G$ and the compact subgroup $K$ reduced to the neutral element e. Before exhibiting examples of non commutative groups $G$ to which Gelfand's theorem applies, let us show how harmonic analysis (i.e. the theory of commutative locally compact groups) 
generalizes to Gelfand pairs.

Applying the general theory of commutative Banach algebras, one first looks for the characters of the commutative algebra $L^{I}(K \backslash G / K)$; they can be written in a unique manner.

$$
f \mapsto \zeta_{\omega}(f)=\int_{G} f(x) \omega(x) d m_{G}(x)
$$

where the complex function $\omega$ is uniformly continuous (both left and right), such that $|\omega(x)| \leq \omega(e)=1$, and $\omega(t x)=\omega(x t)=\omega(x)$ for $t \in K$ and $x \in G$. These functions are called the (zonal) spherical functions for the Gelfand pair (G,K) ; for any such function, the complex conjugate $\bar{\omega}$ and the function $\stackrel{v}{\omega}(\mathbf{x})=\omega\left(\mathrm{x}^{-1}\right)$ are also spherical functions. If $G$ is commutative and $K=\{e\}$, spherical functions are the characters of $\mathrm{G}$, continuous homomorphisms of $\mathrm{G}$ into the group $\underline{U}$ of complex numbers of absolute value 1. The following properties generalize those of characters of commutative locally compact groups :

I) For bounded continuous functions on $G$, invariant by left and right translations by elements of $\mathrm{K}$, the following are equivalent :

a) $\omega$ is a spherical function :

b) $\int_{K} \omega(x t y) d m_{K}(t)=\omega(x) \omega(y)$ for $x, y$ in $G\left(m_{K}\right.$ is the Haar measure on $K$ with total mass 1) ;

c) $\omega(e)=1$ and $f * \omega=\lambda_{f} \omega$ for some scalar $\lambda_{f} \in \underline{C}$, for all $f \in \mathcal{K}(K \backslash G / K)$ (when $G$ is commutative and $\mathrm{K}=\{\mathrm{e}\}$, equation $\mathrm{b}$ ) becomes $\omega(\mathrm{xy})=\omega(\mathrm{x}) \omega(\mathrm{y})$ and, in $\mathrm{c})$, $\left.\lambda_{f}=\int_{G} f(t) \overline{\omega(t)} d m_{G}(t)\right)$.

II) The set $\mathrm{S}(\mathrm{G} / \mathrm{K})$ of spherical functions is locally compact for the compact-open topology, which coincides on that set with the weak* topology of $L^{\infty}$ (G) (when $\omega$ is identified with the character $\zeta_{\omega}$ of $\left.L^{1}(K \backslash G / K)\right)$. The mapping $(\omega, x) \mapsto \omega(x)$ of $\mathrm{G} \times \mathrm{S}(\mathrm{G} / \mathrm{K})$ into $\underline{\mathrm{C}}$ is continuous, and every compact subset of $\mathrm{S}(\mathrm{G} / \mathrm{K})$ is equicontinuous . (When $G$ is commutative and $K=\{e\}, S(G / K)$ is the dual group $\hat{G}$ of $G$, but of course in general $\mathrm{S}(\mathrm{G} / \mathrm{K})$ has no group structure). 
III) To each function $f \in L^{1}(G)$ is associated its Fourier transform, which is a function on the space $S(G / K)$, defined by

$$
\mathcal{F}_{f}: \omega \mapsto \int_{G} f(x) \omega\left(x^{-1}\right) d_{G}(x) \quad ;
$$

it is continuous and tends to 0 at infinity. Furthermore, if $f, g$ are any two functions in $L^{1}(K \backslash G / K)$, one has

$$
\begin{aligned}
\left|\mathcal{F}_{f}(\omega)\right| \leq & \int_{G}|f(x)| d_{G}(x)=N_{1}(f) \text { for all } \omega \in S(G / K) ; \\
& \mathcal{F}(f * g)=\mathcal{F}_{f} . \mathcal{F}_{g} .
\end{aligned}
$$

IV) An important notion in the theory of representations of locally compact groups is that of functions of positive type : they are (complex valued) bounded continuous functions $x \mapsto p(x)$ on the group $G$, such that, for any finite subset $\left\{s_{1}, s_{2}, \ldots, s_{n}\right\}$ in $G$, one has

$$
\sum_{j, k} p\left(s_{j}^{-1} s_{k}\right) \bar{\zeta}_{j} \zeta_{k} \geq 0
$$

for all systems $\left\{\zeta_{1}, \zeta_{2}, \ldots, \zeta_{n}\right\}$ of complex numbers. An equivalent condition is that, for any function $g \in \mathcal{K}(G)$, one has

$$
\int_{G} p(x)\left(\frac{v}{g} * g\right)(x) d m_{G}(x) \geq 0 .
$$

It is immediate to verify that for a locally compact commutative group $G$, the characters of $G$ are functions of positive type. But for a Gelfand pair $(G, K)$, it is not true in general that spherical functions are functions of positive type; one is thus led to consider in $\mathrm{S}(\mathrm{G} / \mathrm{K})$, the closed subspace $\mathrm{Z}(\mathrm{G} / \mathrm{K})$ of spherical functions of positive type; it is on that space that one obtains the most interesting results, and in particular the closest generalization of commutative harmonic analysis.

V) In the first place, for a function of positive type $p$ on a non discrete group $G$, the sesquilinear form

$$
\left.(h, g) \mapsto \int_{G} \stackrel{\check{h}}{(\underline{h}} \star g\right)(x) p(x) d m_{G}(x)
$$


defines on $L^{1}(G)$ a structure of preHilbert space, which, by passage to quotient and completion, yields a Hilbert space $E_{p}$; the left translation by an element $s \in G$ extends to a unitary transformation $\underline{U}(s)$ of $E_{p}$; $s i \rightarrow \underline{U}(s)$ is then a unitary representation of $G$ into $E_{p}$, and there is in $E_{p}$ a vector $x_{0}$ such that

$$
p(s)=\left(\underline{U}(s) \cdot x_{o} \mid x_{0}\right) \quad \text { for all } s \in G \text {. }
$$

In particular, if $(G, K)$ is a Gelfand pair, and $\omega$ a spherical function of positive type for $(G, K)$, there is in this manner a unitary representation $U_{\omega}$ of $G$ in a Hilbert space $E_{\omega}$ associated to $\omega$; a remarkable property is that $\frac{U}{\omega}$ is irreducible and that its restriction to $\mathrm{K}$ contains exactly once the trivial representation of $\mathrm{K}$; furthermore, conversely, every irreducible unitary representation of $G$ in a Hilbert space, the restriction of which to $\mathrm{K}$ contains (at least once) the trivial representation of $\mathrm{k}$, is equivalent to one and only one representation $\underline{U}_{\omega}$ for a function $\omega \in Z(G / K)$. VI) The concept of function of positive type is a special case of the notion of complex measure $\mu$ of positive type on $G$ : it is defined by the condition that, for any $g \in K(G)$, one has

$$
\int_{G}\left(\frac{v}{g} * g\right)(x) d \mu(x) \geq 0,
$$

and therefore the functions of positive type $p$ are those such that $p \cdot m_{G}$ is a measure of positive type. The same construction as in $\mathrm{V}$ ) (with $\mu$ replacing $\mathrm{p} . \mathrm{m}_{\mathrm{G}}$ ) yields again a Hilbert space $E_{\mu}$, in which one considers the closed subspace $H_{\mu}$, the closure of the image of $\kappa(K \backslash G / K)$. If $\pi: K(K \backslash G / K) \rightarrow H_{\mu}$ is the natural mapping, one may write, for $f, g$ in' $\chi(K \backslash G / K), \pi\left(f_{*} g\right)={ }_{-\mu}(f) . \pi(g)$ and $\underline{V}_{\mu}$ is a continuous homomorphism of the commutative algebra $\not(K \backslash G / K)$ into the algebra $\mathcal{L}\left(H_{\mu}\right)$ of continuous endomorphisms of $\mathrm{H}_{\mu}$. To this homomorphism one may apply a fundamental theorem of spectral theory, known as the Plancherel-Godement theorem: it shows that, on the locally compact space $\mathrm{Z}(\mathrm{G} / \mathrm{K})$, there is a unique positive measure $\mu^{\Delta}$ such that, for every function $g \in K(K \backslash G / K)$, the cotransform $\mathcal{F} g=\mathcal{F} \frac{v}{g}$ of $g$ belongs to 
$L^{2}\left(\mu^{\Delta}\right)$, and for any two functions $f, g$ of $\kappa(K \backslash G / K)$, one has

$$
\underline{\underline{v}}(\mathrm{~h} * g)=\int_{Z} \overline{\mathcal{F}}_{g}(\omega) \overline{\overline{\mathfrak{F}}}_{h}(\omega) \mathrm{d} \mu_{1}^{\Delta}(\omega),
$$

and $H_{\mu}$ is naturally isomorphic to $L^{2}\left(\mu^{\Delta}\right)$. One says that $\mu^{\Delta}$ is the Plancherel transform of the measure $\mu$ of positive type. For instance, it is easily seen that the Dirac measure $\varepsilon_{e}$ at the neutral element e of $G$ is a measure of positive type; its Plancherel transform $\varepsilon_{\mathrm{e}}^{\Delta}$ is written $\mathrm{m}_{\mathrm{Z}}$ and called the canonical measure on $Z(G / K)$; for $\mu=\varepsilon_{e}$, relation (12) gives

$$
\int_{G} g(x) \overline{h(x)} d_{G}(x)=\int \mathcal{Z} g(\omega) \overline{\mathcal{J}_{h}(\omega)} d m_{Z}(\omega) .
$$

If $G$ is commutative and $K=\{e\}, m_{Z}$ is the Haar measure on the dual $\hat{G}$ associated with $m_{G}$, and (13) is the usual Plancherel formula. Relation (13) shows that the Fourier transform $f \mapsto \rightarrow \mathcal{F}$ extends to an $\underline{\text { isomorphism }}$ of the Hilbert space $L^{2}(K \backslash G / K)$ (the closure of $\mathcal{H}(K \backslash G / K)$ in $L^{2}(G)$ ) onto the Hilbert space $L^{2}\left(Z(G / K), m_{Z}\right)$, the generalization of the well-known isomorphism of $L^{2}(G)$ onto $L^{2}(\hat{G})$ in the commutative case.

For any spherical function $\omega \in Z(G / K)$ of positive type, $\omega . m_{G}$ is a measure of positive type and its Plancherel transform is

$$
\left(\omega \cdot m_{G}\right){ }^{\Delta}=\varepsilon_{\omega}
$$

the Dirac measure on $\mathrm{Z}(\mathrm{G} / \mathrm{K})$ at the point $\omega$.

Finally, it may be shown that any bounded measure $\mu$ on $G$ is a linear combination of measures of positive type, and therefore 1ts Plancherel transform $\mu^{\Delta}$ is defined; furthermore, $\mu^{\Delta}$ has a density with respect to the canonical measure $m_{Z}$, which is continuous and bounded, and written $\mathcal{F}_{\mu}$; it is given by the formula

$$
\left(\Im_{\mu}\right)(\omega)=\int_{G} \omega\left(x^{-1}\right) d \mu(x),
$$

which extends to bounded measures the definition (4) of the Fourier transform. When $G$ is commutative and $\mathrm{K}=\{e\}$, relation (15) is written

$$
\mathcal{F} \mu(\hat{x})=\int_{G} \overline{\langle x, \hat{x}\rangle} d \mu(x)
$$


and for a bounded measure $\mu$ on $G$, relation (12) is written

$$
\int_{G}(g * h)(x) d \mu(x)=\int_{\hat{G}} \overline{\mathcal{F}} g(\hat{x}) \overline{\mathcal{F} h}(\hat{x}) \mathscr{F}_{\mu}(\hat{x}) d_{\hat{G}}(\hat{x})
$$

(generalized Plancherel theorem).

VII) When $G$ is a unimodular connected Lie group, it may be shown that spherical functions corresponding to a Gelfand pair $(G, K)$ are of class $C^{\infty}$ and are eigenvectors of all differential operators which are invariant under left translations by elements of $G$ and right translations by elements of $K$. On semisimple Lie groups, this implies that spherical functions are analytic, because among these invariant operators there are always in that case elliptic ones.

The fundamental examples of spherical functions: There are three main types of Gelfand pairs $(G, K)$ with noncommutative groups $G$, each giving rise to spherical functions among which are many of the "special functions" of Analysis.

A) G is a linear semisimple compact connected Lie group; then, for any involutive automorphism $\sigma$ of $G$, the conditions of Gelfand's theorem are satisfied; all these automorphisms have been explicitly determined by E. Cartan. In this case, al1 spherical functions are of positive type, and the space $S(G / K)$ is discrete.

The most interesting example is given by the group $\mathrm{G}=\underline{\mathrm{S}} 0(\mathrm{n}+1)$ of rotations in the Euclidean space $\underline{R}^{n+1}$, and the subgroup $K=\underline{S O}(n) ; G / K$ is identified with the sphere $\underline{S}_{n}$, K being the subgroup which leaves invariant the first vector $e_{0}$ of the canonical basis of $\underline{\mathrm{R}}^{\mathrm{n}+1}$. As $\mathrm{G}$ is compact, $\mathrm{L}^{2}(\mathrm{G})$ is an algebra (for the convolution product), and so is the closure $L^{2}(K \backslash G / K)$ of $h(K \backslash G / K)$ in $L^{2}(G)$, which is therefore commutative by Gelfand's theorem. The functions of $L^{2}(K \backslash G / K)$ can be identified to the functions in $L^{2}\left(\underline{S}_{n}\right)$ which only depend on one variable, namely the angle $\theta$ of the variable vector $x \in \underline{S}_{n}$ with the vector $e_{0}$.

For $\mathrm{n} \geq 2$, the space $L^{2}\left(\underline{S}_{n}\right)$ splits into a Hilbert sum of finite dimensional subspaces $E_{m}(m=0,1, \ldots)$ stable under the action of $G: E_{m}$ is exactly the space of the restrictions to $\underline{S}_{n}$ of the harmonic polynomials which are homogeneous of 
degree $m$, and the subrepresentation of $G$ into $E_{m}$ is irreducible. Each $E_{m}$ contains exactly one spherical function $\omega_{m}$; if one writes $\omega_{m}(x)=G_{m, n+1}(\cos \theta)$, the $\mathrm{G}_{\mathrm{m}, \mathrm{n}+1}$ are the Gegenbauer polynomials (the Legendre polynomials for $\mathrm{n}=2$ ) ; they satisfy the differential equation

$$
\left(1-z^{2}\right) y^{\prime \prime}-n z y^{\prime}+m(m+n-1) y=0 \text {. }
$$

B) G is a linear non compact connected semisimple lie group with finite center, and $\mathrm{K}$ is a maximal compact subgroup of $\mathrm{G}$; there is then an involutive automorphism $\sigma$ of $G$ for which $K$ is the group of fixed points, and which satisfies the conditions of Gelfand's theorem. A typical example is given by $G=\underline{S L}(n, \underline{R}), K=\underline{S O}(n)$; $\sigma$ is then the involutive automorphism $\underline{x} \mapsto^{t} \underline{x}^{-1}$ (contragredient matrix).

It may be shown that one may write $G=S K$, where $S$ is a closed solvable subgroup (Iwasawa decomposition). Suppose we know a continuous homomorphism $\alpha$ : $S \rightarrow \underline{C}^{*}$; then one extends $\alpha$ to a continuous function on $G$ by taking $\alpha(s t)=\alpha(s)$ for $s \in S$, $t \in \mathrm{K}$, and one easily checks that the function

$$
\omega(x)=\int_{K} \alpha(t x) d m_{K}(t)
$$

verifies the functional equation

$$
\omega(x) \omega(y)=\int_{K} \omega(x t y) d m_{K}(t)
$$

and therefore is a spherical function if it is bounded (solutions of (20) may be called generalized spherical functions). A deep theorem of Harish-Chandra proves that $\underline{a l l}$ generalized spherical functions relative to the Gelfand pair $(G, K)$ are given by formula (19), and in addition determines explicit1y all homomorphisms $\alpha$, by a detailed study of the Lie algebra of $G$.

The simpliest example consists in the pair $G=\underline{S L}(2, \underline{R}), K=\underline{S O}(2)$; one has then $G=K S=S K$, where $S$ is the solvable group of triangular matrices $\left(\begin{array}{ll}a & b \\ 0 & a\end{array}\right)$ with $a>0$, the decomposition being unique. It is easily shown that the matrices $\underline{X}=\left(\begin{array}{ll}a & b \\ c & d\end{array}\right)$ of a double class relative to $K$ are those for which the number $\operatorname{Tr}\left({ }^{t} \underline{x} \cdot \underline{x}\right)=a^{2}+b^{2}+c^{2}+d^{2}$ has a given value $2 v$ with $v \geq 1$. The functions of $C(K \backslash G / K)$ are therefore the functions 
$f\left(\frac{1}{2}\left(a^{2}+b^{2}+c^{2}+d^{2}\right)\right)$, where $f$ is continuous on the half-1ine $[1, \infty[$. One then finds for the generalized spherical functions

$$
P_{\rho}(v)=\frac{1}{2 \pi} \int_{0}^{2 \pi}\left(v+\sqrt{v^{2}-1} \cos \phi\right)^{\rho} d \phi
$$

where $\rho$ is any complex number ; they are the Legendre functions of index $\rho$, and the corresponding functional equation (20) is

$$
P_{\rho}(\operatorname{ch~} t) P_{\rho}(\operatorname{ch~} u)=\frac{1}{2 \pi} \int_{0}^{2 \pi} P_{\rho}(\operatorname{ch} t \operatorname{ch~u+sht} \operatorname{sh} u \cos \phi) d \phi
$$

$(t, u$ arbitrary real numbers).

C) The third case consists of a unimodular group G containing a normal commutative group A with no elements of order 2, and a (non normal) compact subgroup $\mathrm{K}$, such that the mapping $(t, s) \mapsto t s$ is a diffeomorphism of the manifold $K \times A$ onto $G$; one then checks that $\sigma: t s \mid \rightarrow t s^{-1}$ is an involution having the properties required in Gelfand's theorem, so that $(G, K)$ is a Gelfand pair. One then starts from a continuous homomorphism $\alpha: A \rightarrow \underline{C}^{*}$, and for $x=t s$ with $t \in K, s \in A$, one defines

$$
\omega(x)=\int_{K^{\alpha}}\left(u^{-1}\right) u^{-1} m_{K}(u)
$$

It is easily verified that $\omega$ satisfies the functional equation (20), and is thus a generalized spherical function ; furthermore, it can be shown that all generalized spherical functions are obtained in this manner.

The typical example here is the group $G$ of isometries of the Euclidean plane $\underline{\mathrm{R}}^{2}$ preserving orientation; it can be identified with the group of matrices

$$
\left(\begin{array}{ccc}
\cos \theta & \sin \theta & x \\
-\sin \theta & \cos \theta & y \\
0 & 0 & 1
\end{array}\right)
$$

acting by multiplication on vectors of $\underline{\mathrm{R}}^{2}$ represented by column vectors

$$
\left(\begin{array}{l}
a \\
b \\
1
\end{array}\right)
$$

The normal subgroup $A$ is here the group of translations (corresponding to matrices with $\theta=0$ ), and $K$ the group of rotations (corresponding to $x=y=0$ ). The 
homogeneous space $\mathrm{G} / \mathrm{K}$ is here Identified with $\underline{\mathrm{R}}^{2}$, and the double classes KsK are identified with the orbits of $K$ in $\underline{R}^{2}$, 1.e. the circles $x^{2}+y^{2}-x^{2}$; continuous functions on $K \backslash G / K$ are thus 1dentified with functions $\psi\left(\left(x^{2}+y^{2}\right)^{1 / 2}\right)$, where $\psi$ is continuous on the interval $\left[0,+\infty\left[\right.\right.$. The continuous homomorphisms $\alpha: A \rightarrow \underline{C}^{*}$ are here the exponent1al. $(x, y)-\operatorname{axp}(\lambda x+\mu y)$ with $\lambda, \mu$ arbitrary complex numbers. Formula (23) therefore 1dentifies here the solutions of (20) to the continuous functions on $[0,+\infty[$ given by

$$
\psi(r)=\frac{1}{2 \pi} \int_{0}^{2 \pi} \exp (r(\lambda \cos \phi+\mu \sin \phi)) d \phi \quad
$$

When one takes $\lambda=0, \mu=1$, one gets the Bessel function $J_{0}$, which is bounded, hence gives a spherical function.

\section{REFERENCES}

1. Dieudonné, J., Treatise on Analysis, Volume V (Chapter XXI) and Volume VI (Chapter XXII) Academic Press, 1977.

2. Vilenkin, N.J., Special Functions and the Theory of Group Representations. Translations of Mathematical monographs, Volume 22, American Mathematical Society, Providence, 1968.

3. Gangolli, R., Symmetric Spaces, (Short courses presented at Washington University, edited by W. Boothby and G. Weiss) Dekker, New York, 1972.

4. Gelfand, I., Graev, M., and Pyatetskii-Shapiro, I., Representation Theory and Automorphic Functions, Saunders, Philadelphia-London-Toronto, 1969.

5. Helgason, S., Differential Geometry and Symmetric Spaces, Academic Press, 1962. 


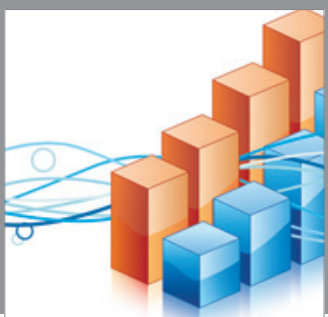

Advances in

Operations Research

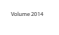

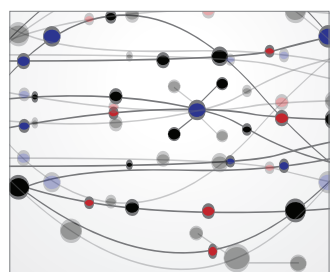

\section{The Scientific} World Journal
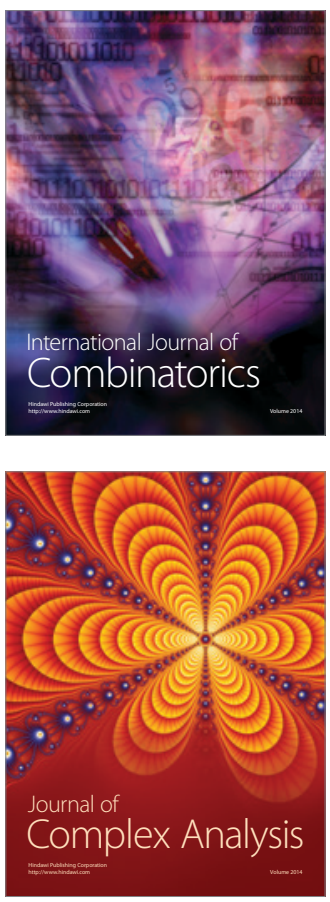

International Journal of

Mathematics and

Mathematical

Sciences
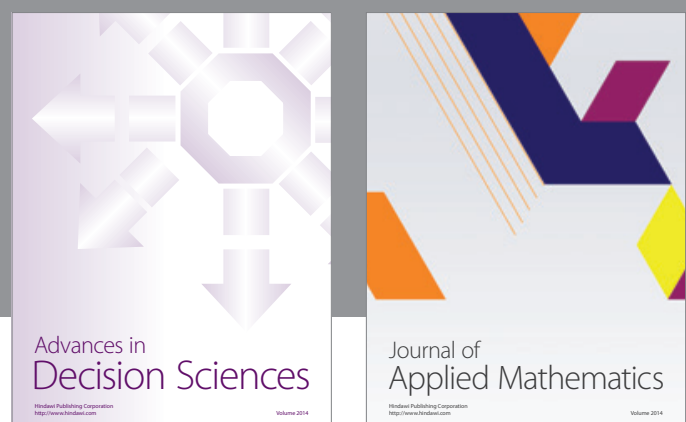

Journal of

Applied Mathematics
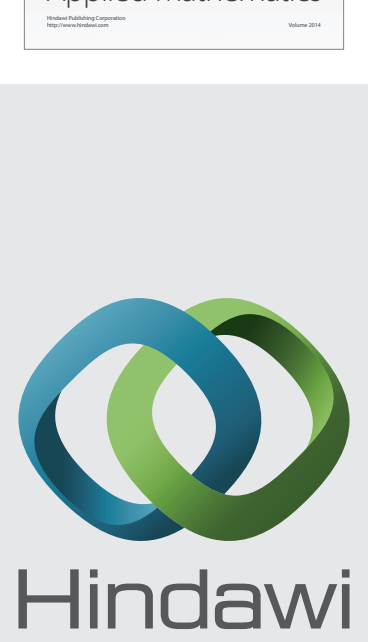

Submit your manuscripts at http://www.hindawi.com
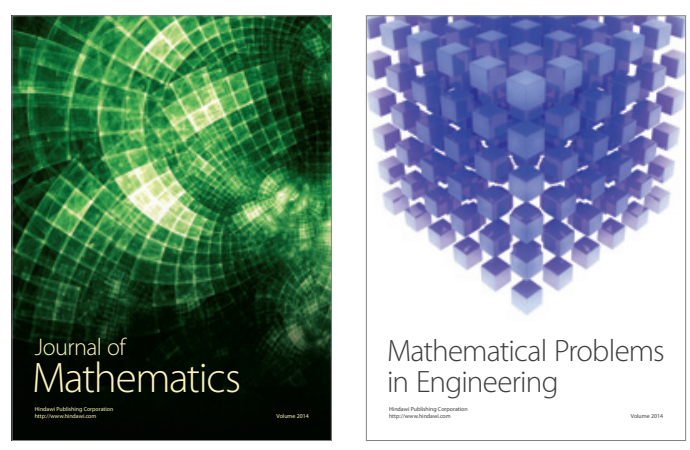

Mathematical Problems in Engineering
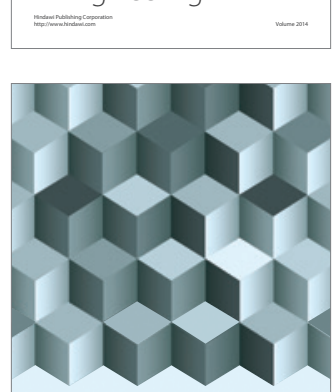

Journal of

Function Spaces
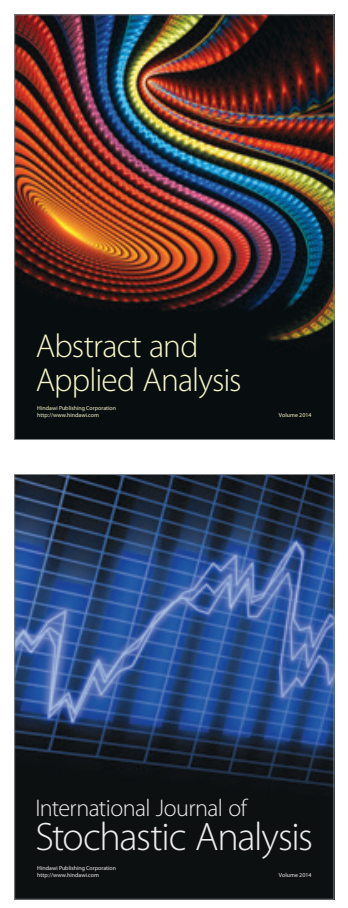

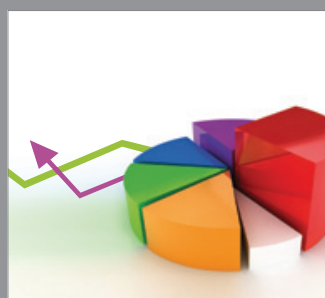

ournal of

Probability and Statistics

Promensencen
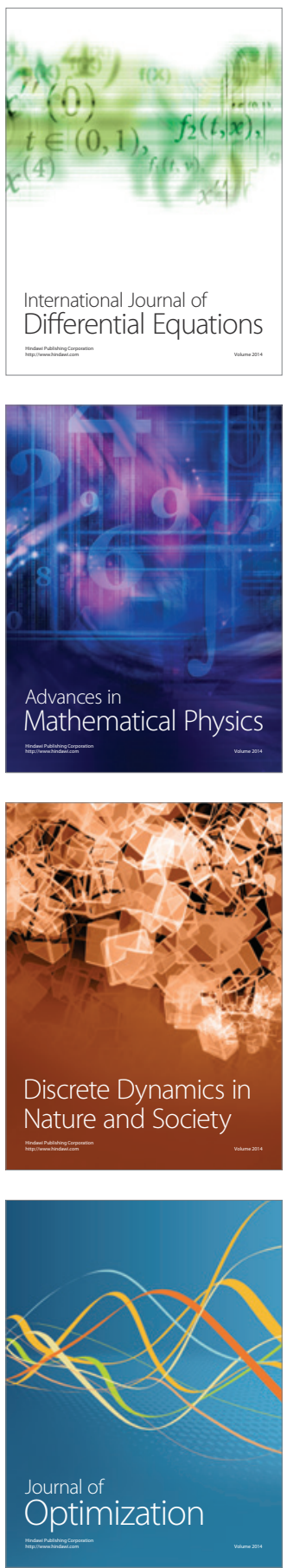